\title{
The diagnostic conundrum of Riehl melanosis and other facial pigmentary disorders: a case report with overlapping clinical, dermoscopic, and histopathological features
}

\author{
Irma Bernadette S. Sitohang ${ }^{1}$, Rizky Lendl Prayogo ${ }^{1}$, Rahadi Rihatmadja ${ }^{1}$, Sondang P. Sirait ${ }^{1}$ \\ ${ }^{1}$ Department of Dermatology and Venereology, Faculty of Medicine, Universitas Indonesia, Dr. Cipto Mangunkusumo Hospital, Jakarta, Indonesia.
}

\begin{abstract}
Riehl melanosis (RM) is a form of pigmented contact dermatitis that often poses a diagnostic challenge due to overlaps in its clinical, dermoscopic, and histopathological features with other pigmentary disorders. This report highlights significant findings and the proper approach for diagnosis. We present the case of a 47-year-old female with progressive facial hyperpigmentation (irregular, blotchy, grayish patches on the forehead, cheeks, and around the mouth) that acknowledged applying a lightening product before her complaint. Dermoscopy revealed brownish-gray dots and pseudonetwork pigmentation. The histopathological findings were comprised of hypergranulosis, interface dermatitis, and pigment incontinence. The patient's history and clinical, dermoscopic, and histopathological examination results showed features consistent with-but not exclusive to-RM, such that it was not possible to rule out lichen planus pigmentosus (LPP) and ashy dermatosis (AD). We finally diagnosed the patient with RM and treated her with medications consisting of alpha hydroxy acid, tretinoin, hydroquinone, and sunscreen. Patch testing might help because higher positivity rates were found in RM patients compared to LPP and AD. However, positive patch testing favors RM, and, regardless of diagnosis, provides a basis for substance avoidance in the treatment plan.
\end{abstract}

Keywords: Riehl melanosis, diagnosis, dermoscopy, histopathology, case report

Received: 22 December 2019| Returned for modification: 10 February 2020 | Accepted: 26 February 2020

\section{Introduction}

Riehl melanosis (RM) is a pigmentary disorder first described in 1917 during the First World War (1). Riehl could not determine the exact etiology of this disorder, but he suspected the role of the abnormal wartime diet (1). It manifests as facial hyperpigmentation, mainly affecting the forehead, zygomatic, and/or temporal regions $(2,3)$. Later, in 1973, Nakayama et al. observed the same conditions in Japan and hypothesized that it involved exposure to contact substances $(1,4)$. It is now known that sensitizing chemicals in cosmetics are the most common cause $(3,4)$. Therefore, it is suggested that it is synonymous with pigmented cosmetic dermatitis (PCD) (4).

In this report, we further consider RM and PCD to be synonymous and that they may be used interchangeably. RM/PCD often poses a diagnostic challenge due to overlaps in its clinical, dermoscopic, and histopathological features with other pigmentary disorders, notably lichen planus pigmentosus (LPP) and ashy dermatosis (AD) (4). Thus, the existence of these disorders as distinct entities or as variants of the same process has been a topic of perpetual debate (4). Because there has been no detailed attempt at discussing treatment modalities (3), this report highlights essential findings and a proper diagnostic approach for these disorders, especially RM/PCD.

\section{Case report}

A 47-year-old female presented with a yearlong history of progressive facial hyperpigmentation. Three months before her complaint, she acknowledged applying a lightening product contain- ing octyl methoxycinnamate, a potential contact sensitizer. The hyperpigmented rash appeared initially on her chin a year previously. Within 6 months, it had already spread to her forehead and cheeks, and around her mouth. She then consulted our outpatient clinic to have the abnormal pigmentation removed. Upon physical examination, we observed numerous, discrete to confluent, fine, brownish-gray macules, some of which were arranged in a reticulated pattern on the forehead and cheeks, and around the mouth (Fig. 1). Dermoscopy showed diffuse erythema, telangiectasia, multiple brown and gray dots/granules, pseudonetwork pigmentation, and a perifollicular whitish halo (Fig. 2). Histopathological examination using hematoxylin and eosin staining showed basket-weave stratum corneum, hypergranulosis, colloid bodies, dense inflammatory infiltrates within the dermo-epidermal junction, increased melanin deposition, pigment incontinence, and melanophages (Fig. 3). Based on these findings, other abnormal dermal pigmentations such as melasma and ochronosis could be excluded. However, overlapping clinical, dermoscopic, and histopathological features presented in our patient with RM/PCD, LPP, and AD. We finally diagnosed the patient with RM/PCD and treated her with medications consisting of alpha hydroxy acid $10 \%$ twice daily, sunscreen every 3 hours, tretinoin $0.05 \%$, and hydroquinone $4 \%$ once daily at night for an initial 2-week period. After 2 weeks, she noticed a slight improvement and did not experience any adverse events. We planned to perform a patch test to determine the causative allergen. Unfortunately, the patient moved to another island, could not be contacted for a scheduled appointment, and was lost to follow-up. Further explanation regarding the diagnosis is elaborated in the discussion. 


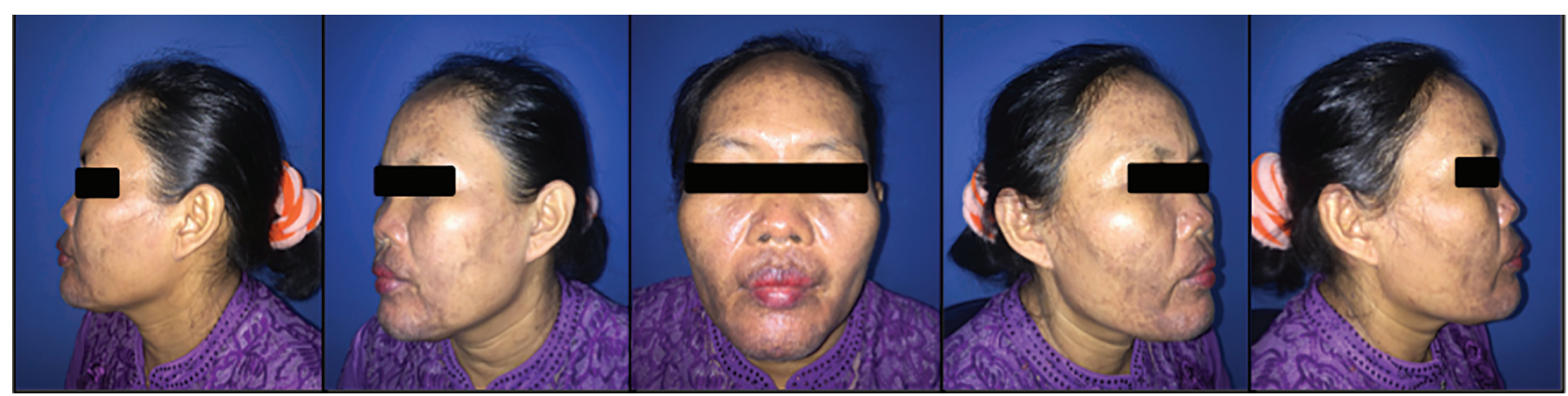

Figure 1 | Clinical findings: brownish-gray macules on the forehead and cheeks, and around the mouth.
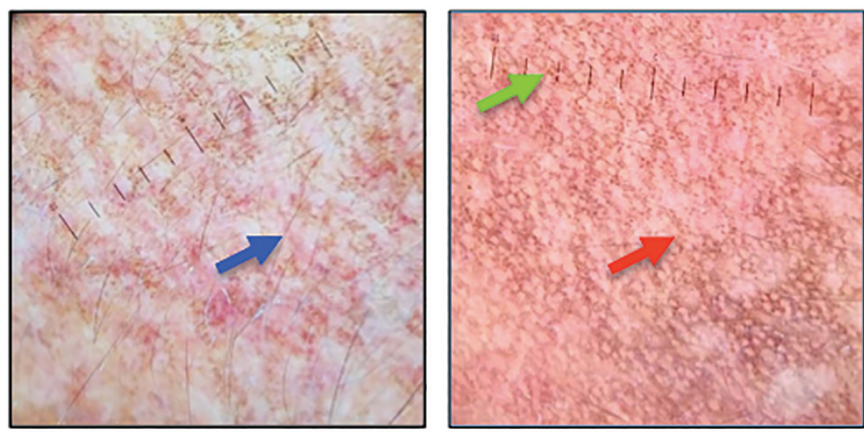

Figure 2 | Dermoscopic findings: diffuse erythema, telangiectasia (blue arrow), multiple brown and gray dots/granules (green arrow), pseudonetwork pigmentation (red arrow), and perifollicular whitish halo.
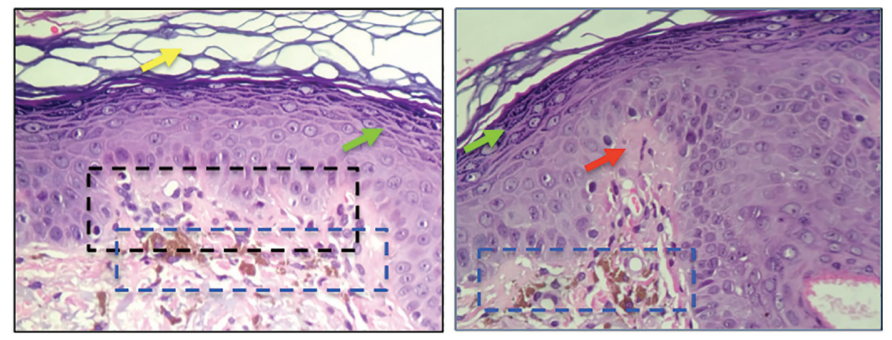

Figure 3 | Histopathological findings (H\&E, 400x): basket-weave stratum corneum (yellow arrow), hypergranulosis (green arrows), colloid bodies (red arrow), dense inflammatory infiltrates within the dermo-epidermal junction (black square), and increased melanin deposition (blue squares).

\section{Discussion}

$\mathrm{RM} / \mathrm{PCD}$ is a form of contact dermatitis, mainly affecting the forehead, zygomatic, and/or temporal regions (2). It has been found in dark-complexioned people (2). The diagnosis of RM/PCD is not always simple or straightforward because it shows variable clinical features (2). Dermoscopy has been shown to be useful for accurately diagnosing some pigmented skin lesions (2). In some cases, biopsy and histopathological examination may be essential for making a definite diagnosis (2). LPP and AD share some similar clinical, dermoscopic, and histopathological features with RM/PCD (4). Although they do not share all features, Bhutani considered $\mathrm{AD}$ and RM/PCD to be the same disease as LPP $(5,6)$. To avoid the controversy, a global consensus on the terminology of various morphologies of acquired macular pigmentation of uncertain etiology was held after the 22nd International Pigment Cell Conference in Singapore (3). Thirty-nine experts representing 18 countries participated in the deliberations to define each term and distinguish RM/PCD, LPP, and AD (3). This report highlights the critical clinical, dermoscopic, and histopathological features of RM/PCD found in our patient and therefore makes a proper diagnostic approach.

\section{Clinical features}

Our patient showed facial pigmentation with numerous discrete to confluent brownish-gray macules, some arranged in a reticulated pattern with well- to ill-defined borders. These characteristics, however, may be present in RM/PCD, LPP, and AD (4). The absence of an erythematous hue in our patient makes the diagnosis of RM/PCD and LPP highly probable because it is more commonly found in $\mathrm{AD}$ (4). In addition, the global consensus differentiates $\mathrm{RM} / \mathrm{PCD}$, LPP, and AD based on the size of the lesions and predilection areas (3). RM/PCD is characterized by numerous fine (a few millimeters in size) or reticulate macules on the face, neck, and upper chest (3). LPP gives the appearance of large $(>5 \mathrm{~cm})$ and small $(0.5-5 \mathrm{~cm})$ pigmentation involving the head, neck, and flexural areas (3), whereas AD lesions consist only of large $(>5 \mathrm{~cm})$ hyperpigmented macules on the trunk (3). Unlike LPP and AD, RM/PCD does not consist of larger macules, nor does it affect the flexures and trunk. Despite some similarities, the clinical features in our patient were more consistent with RM/PCD. We concluded that the size of the lesions and predilection areas were crucial for clinically differentiating RM/PCD, LPP, and AD.

\section{Dermoscopic features}

The dermoscopic features found in our patient were multiple brownish-gray dots and granules, pseudonetwork pigmentation, a perifollicular whitish halo, diffuse erythema, and telangiectasia. Dots or granules can be present in RM/PCD, LPP, and AD (4). Pseudonetwork pigmentation, a perifollicular whitish halo, and erythema may be present in RM/PCD and LPP (4). Based on these characteristics, we concluded that there was no single pathognomonic dermoscopic feature that exclusively belongs to one disorder.

\section{Histopathological features}

From the histopathological examination, hypergranulosis, basal cell damage, colloid bodies, lichenoid infiltrate, and pigment incontinence were found in our patient. Hypergranulosis is usually present in LPP but is absent in RM/PCD and AD (4). Basal cell damage and colloid bodies are usually (or may be) present in RM/ PCD, LPP, and AD (4). Lichenoid infiltrate is absent in RM/PCD and AD, and usually absent in LPP (4). Finally, pigment incontinence is present in all three disorders (4). It remains challenging to differentiate these disorders based only on the histopathological features because there were no histopathological features that appear exclusive to one disorder. Despite the presence of hypergranulosis, we finally diagnosed our patient with RM/PCD because the clinical features were more consistent with it. 


\section{Patch test}

Tienthavorn et al. (7) compared provisional diagnosis and actual cases with a positive patch test result by diagnosis. Those that were diagnosed with PCD had positive patch tests in $80 \%$ of cases, higher than LPP and AD (7). We were interested in performing a patch test in our patient because of this association between PCD and a positive patch test result. If a specific relevant contact allergy is demonstrated, the disorder may be better labeled as PCD (3). We finally diagnosed our patient with RM and treated her with medications consisting of alpha hydroxy acid 10\%, sunscreen, tretinoin $0.05 \%$, and hydroquinone $4 \%$.

Despite the lack of patch test results, this case report has some significant strengths. It highlights the essential findings of RM/ PCD, LPP, and AD. All similarities and differences were thoroughly discussed based on the latest consensus and references. We believe that it can serve as a basis for a proper diagnostic approach to these disorders, especially RM/PCD.

\section{Conclusions}

RM/PCD, LPP, and AD are considered distinct entities. They share overlapping clinical, dermoscopic, and histopathological features. RM/PCD is characterized by extensive fine/reticulate pigmentation on the face, neck, and upper chest. PCD is the more appropriate term if a patch test points to a specific contact allergy; otherwise, it is better designated as RM. Furthermore, positive patch test results provide the basis for substance avoidance in the treatment plan.

\section{References}

1. Nakayama H. Pigmented contact dermatitis and chemical depigmentation. In: Johansen JD, Frosch PJ, Lepoittevin J-P, editors. Contact dermatitis. 5th ed. New York: Springer; 2012. p. 377-93.

2. Wang L, Xu AE. Four views of Riehl's melanosis: clinical appearance, dermoscopy, confocal microscopy and histopathology. J Eur Acad Dermatol Venereol. 2014;28:1199-206.

3. Kumarasinghe SPW, Pandya A, Chandran V, Rodrigues M, Dlova NC, Kang HY, et al. A global consensus statement on ashy dermatosis, erythema dyschromicum perstans, lichen planus pigmentosus, idiopathic eruptive macular pigmentation, and Riehl's melanosis. Int J Dermatol. 2019;58:263-72.

4. Gupta V, Sharma VK. Ashy dermatosis, lichen planus pigmentosus and pigmented cosmetic dermatitis: are we splitting the hair? Indian J Dermatol Venereol Leprol. 2018;84:470-4.

5. Bhutani LK. Ashy dermatosis or lichen planus pigmentosus: what is in a name? Arch Dermatol. 1986;122:133.

6. Bhutani LK. Pigmented contact dermatitis vs lichen planus pigmentosus. Int J Dermatol. 1977;16:860-2.

7. Thienthavorn T, Tresukosol P, Sudtikoonaseth P. Patch testing and histopathology in Thai patients with hyperpigmentation due to erythema dyschromicum perstans, lichen planus pigmentosus, and pigmented contact dermatitis. Asian Pac J Allergy Immunol. 2014;32:185-92. 